Centre, Sydney University, Sydney, Australia; '5Journalism and Media Research Centre UNSW, Sydney, Australia; ' 6 Sydney University Discipline of Paediatrics and Child Health The Children's Hospital at Westmead, Sydney, Australia

Introduction There is considerable public concern that new media (including the Internet and mobile phones) could be exposing young people to high levels of sexual content and may impact risky behaviour and/or risk for sexually transmissible infections.

Methods The review was conducted in accordance with the PRISMA guidelines. Medline, EMBASE and PsychINFO were searched to the end of August 2012. Articles were included if they described the statistical association between exposure to sexual content in new media (viewing or engaging) and sexual attitudes or behaviours in young people (defined as $<25$ years).

Results There were 3834 articles identified, and five met the inclusion criteria: all were cross-sectional designs (four convenience samples), with the largest study containing 6054 participants. In four studies the exposure was viewing sexually explicit websites (SEW); a range of outcomes were assessed however each outcome variable was only measured in one or two of the included studies. Viewing SEW was significantly associated with: first intercourse $<15$ years of age, ever having a sexual partner, $>1$ partner in last 3 months, multiple lifetime partners, unprotected sex at last sex, drug and alcohol use at last sex, acceptance of casual sexual relationships, having casual sexual relationships, notions of women as sex objects, and approval of extra-marital sex. "Sexting" (sending or receiving sexual texts) was the exposure in the fifth study and was associated with ever having unprotected sex.

Conclusion The relationship between SEW and sexual behaviours and attitudes was inconsistent. Engagement with sexual media appeared to be associated with markers of higher sexual interest and/or activity, but study size, methodological approach and inconsistencies in outcome measures prevented us from drawing conclusions regarding causality. No study explored associations with positive aspects of sexual development (e.g. sexual communication, sexual assertiveness, relationship quality). Further research in this emerging area is needed.

\section{P4.025 CONCEPTUALIZATION OF ANAL SEX BEHAVIOUR WITHIN SEXUAL PARTNER RELATIONSHIPS FOR MODIFICATION OF EVIDENCE-BASED ADOLESCENT SEXUAL HEALTH PROMOTION INTERVENTIONS}

doi:10.1136/sextrans-2013-051184.0923

IJ D Champion, ${ }^{2} \mathrm{C}$ Roye. ${ }^{1}$ The University of Texas at Austin, Austin, TX, United States, 2Hunter, CUNY, New York, NY, United States

Background Understanding of the context of anal sex behaviour among Black and Hispanic adolescent women with a history of sexually transmitted infection (STI/HIV) has public health implications for health promotion and risk reduction behavioural interventions.

Methods African-American ( $\mathrm{n}=94,16.8 \%$ ) and Mexican-American ( $n=465,83.2 \%$ ) women (14-18 years) with histories of sexual risk behaviour, STI or violence, recruited from metropolitan sexual health clinics for participation in a clinical trial for behavioural interventions, completed semi-structured interviews to assess psychosocial and situational factors associated with high sexual risk behaviour, substance use, STI/HIV acquisition, and violence occurrence at study entry.

Results Anal sex varied by ethnicity (24.1\% Mexican-American, $10.6 \%$ African-American, $p=0.004)$ and was associated with more $(p<0.05)$ run-away, school dropout, sexual partners, bisexuality, sex for favours, money or friends with benefits, group sex, substance and alcohol use, sex when high, history of STI, withdrawal for birth control and psychological distress. More (94.3\% vs. $85.1 \%$ ) and higher levels of violence (8.37 vs. 6.38) overall, and by comparisons of sexual and physical violence was identified. Age at first anal sex (15.85 years) with partner age (18.43 years), ever condom use for anal sex (38\%), anal sex past year $(79.5 \%)$, past 6 months $(61.2 \%)$ past 3 months (36.7\%) with limited condom use (40.8\%) was selfreported. Fewer women reporting anal sex could stop having sex with partners having sex with others ( $64.7 \%$ vs. $76.2 \%)$ while more believed it's okay to have a man on the side for more variety/sex (43.9\% vs. $18.3 \%$ ), he fools around so why shouldn't I? (25.8\% vs. $15.1 \%)$ or I get high and lose control (24.2\% vs. $14.5 \%)$.

Conclusions Conceptualization of anal sex behaviour within partner relationships is important for modification of evidence-based sexual health promotion interventions to enhance efficacy for prevention of violence, substance use, unintended pregnancy and STI/ HIV among ethnic minority adolescent women.

\section{P4.026 INDIAN BOARDING SCHOOL EXPERIENCE AND HIV SEXUAL RISK BEHAVIORS AMONG URBAN TWO-SPIRIT AMERICAN INDIAN/ALASKA NATIVES}

doi:10.1136/sextrans-2013-051184.0924

T Evans-Campbell, C R Pearson, K L Walters. University of Washington, Seattle, WA United States

Background As part of a systemic effort of assimilation between the late $19^{\text {th }}$ and mid-20 $0^{\text {th }}$ centuries, thousands of American Indian/ Alaska Native (AIAN) children were placed in residential Indian boarding schools. To understand the impact of forced attendance in Indian boarding schools on HIV risk, we explored sexual risk behaviours, STI diagnosis, and substance misuse among AIAN two-spirit (gay, lesbian, bisexual, and transgender) adults who had attended Indian boarding school as children compared to those with no history of boarding school.

Methods The current investigation was part of a comprehensive multi-site, cross-sectional national health survey of 447 AIAN two-spirit people from seven metropolitan areas in the U.S. To minimise selection bias, multiple sampling strategies were used including targeted, partial network, and respondent-driven sampling (RDS) techniques.

Results Eighty-two (22.9\%) respondents had a history of Indian boarding school attendance. Compared to others, respondents who attended boarding school were more likely to have a diagnosis of alcohol abuse or dependence ( $58.5 \%$ vs. $44.9 \%, p<0.05)$ and reported higher illicit drug use in the past 12 months including: club drugs $(18.3 \%$ vs. $9.6 \%, p<0.05)$, crack-cocaine $(43.9 \%$ vs. $29.6 \%, p<0.01)$, and erectile dysfunction drugs $(7.3 \%$ vs. $2.7 \%, p<0.05)$. Former boarding school attendees also reported more lifetime partners (5.9 vs. $5.4, p<0.05)$, were more likely to have had an STI $(42.7 \%$ vs. $30.7 \%, p<0.05)$, and were more likely to have ever traded sex for drugs, money, or food (56.8\% vs. $33.5 \%, p<0.001)$.

Conclusion Findings from this study provide some of the first data on boarding school experience and HIV risk among an urban twospirit AIAN community sample and underscore the need for increased HIV/STI prevention efforts in AIAN communities. In addition, advanced statistical models are needed that identify mediational pathways to health outcomes and risk behaviours.

\section{P4.027 THE PRACTISE OF ANAL SEX AMONG THE THE FEMALE SEX WORKERS IN THE WESTERN PART OF ASSAM, INDIA}

doi:10.1136/sextrans-2013-051184.0925

A D Sharma. Dermacare Clinic, Bongaigaon, India

Background Unprotected anal sex is associated with the highest risk of transmission of sexually transmitted infections (STIs) and human immunodeficiency virus (HIV). This is due to the delicate nature of the rectal mucosa and anal sphincter. Any from of trauma 